\title{
A portable four-channel cassette recording system for short-term electroencephalogram in the field
}

\author{
JOSEPH S. ALI \\ Bioengineering Branch, Experimental Biology Division, Health Effects Research Laboratory, \\ Environmental Research Center, United States Environmental Protection Agency, \\ Research Triangle Park, North Carolina 27711
}

\begin{abstract}
A portable four-channel electroencephalogram (EEG) cassette recording system has been designed which offers good performance, is simple to construct, and moderate in cost. The recorder is compatible with a commercially available, portable, battery-operated power source, and is therefore usable in the field where line power is unavailable. The system incorporates a small, lightweight, portable, battery-powered oscilloscope which provides monitoring and playback capability. While a few experimental portable EEG recorders have been reported in recent years with more channel capacity or perhaps lighter weight, the engineering details of these units are rarely published and, in any event, would require a substantial effort for most electronics support shops to reproduce. The recording system described here, however, should be within the capability of most electronics support groups to assemble in a very short time and provide good performance.
\end{abstract}

Until recently, instrumentation for recording the EEG in the field has been virtually nonexistent. However, in the last 5 years several promising experimental devices have been reported in which the designs have been specific to the experimental situations at hand. Barlow (1975) has described a 16-channel cassette recorder using a balanced amplitude-modulation timedivision multiplexing technique. This system was developed for clinical use, where many probes are desirable and portability is not of paramount importance. Ives and Woods (1975) have designed a four-channel cassette recorder device for use on ambulatory patients. In this application, weight, size, and recording time were the parameters optimized.

In an effort to study the toxic effects of various pesticides, herbicides, fungicides, and fertilizers on humans, the Environmental Toxicology Division, EPA, has let several contracts to various research and medical institutions having access to people exposed to these potentially toxic agents. Organophosphates, chlorinated hydrocarbons, and carbamates are the classes of substances under investigation. In these studies a recorder is needed which can be taken to the field and operated under its own power so as to record the EEG of a person who has been in the process of applying pesticides. Because of the effort required to schedule subjects, the

The author wishes to thank Mr. E. B. Bailey of Northrop Services Corporation for his capable assistance in construction of the EEG recorder. This report has been reviewed by the Office of Research and Monitoring, EPA, and approved for publication. Approval does not signify that the contents necessarily reflect the views and policies of the Environmental Protection Agency, nor does mention of trade names or commercial products constitute endorsement or recommendation for use. seasonal nature of the work, and the change in pesticides used in different parts of the growing season, monitoring capability of the EEG as it is being recorded is considered essential to insure success at each recording session. This feature will give the EEG technician on location an immediate indication of degradation of signal quality due to failure in electrode contact or other artifacts. For the same reasons, it is considered desirable that the recorder have playback capability. Simplicity of construction and operation, as well as low cost, are also important design considerations.

Since the recorders must be shipped seasonally to coincide with the growing season in various parts of the country, a lightweight, portable system is essential. The recorders are typically shipped to medical or research institutions prior to the season that the substance under investigation will be used locally. A technician charges the power source at the medical facility. He then drives to the farms where the compound of interest is being used and records the EEG of the worker. After a successful recording session is completed, all the cassette tapes are sent to our laboratory at the Research Triangle Park, North Carolina for computer analysis. The recorder may be used at one facility for several months to monitor local workers. At the end of the growing season, the recorder is returned to our laboratory for routine maintenance and check out before being shipped to a new location.

\section{PRINCIPLES OF OPERATION}

This recording system for field use is designed to record three channels of EEG and one channel of ECG on stereo magnetic tape cassettes under battery power 


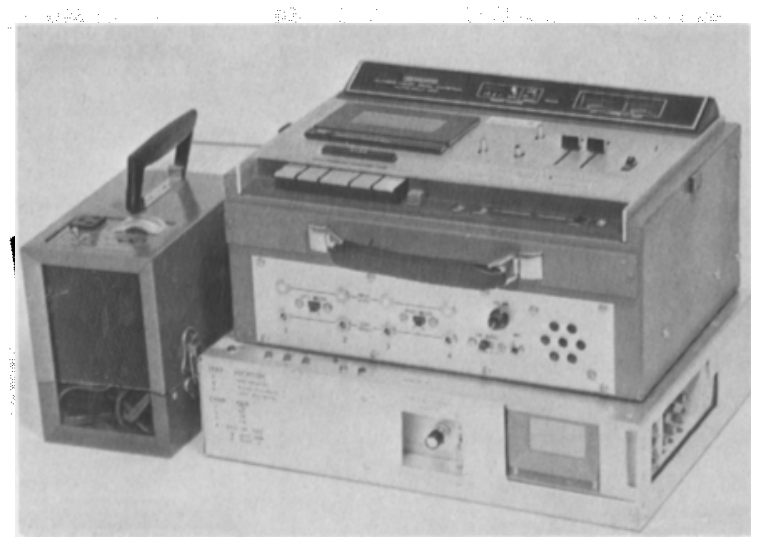

Figure 1. Portable EEG recorder package and power source.

or through ac line power. The entire recording system is contained in two packages: One is a commercially available portable Trav-Electric $117-\mathrm{V}$ ac power source (Model 50-160, Terado Corporation, St. Paul, Minnesota); and the other, a recorder package, contains the analog instrumentation tape recorder, amplifiers, and a monitoring oscilloscope (see Figure 1). The power source has a small self-contained $12-\mathrm{V}$ wet cell battery which is used to generate $117 \mathrm{~V} \mathrm{ac}, 60 \mathrm{~Hz}$ through an inverter. This battery has sufficient capacity to operate the recorder for several hours. The recorder, therefore, can be operated either by direct connection to a conven. tional source of ac power or remotely, in the field, by the use of the power source. The power source can be recharged either from $117-\mathrm{V}$ ac line power or through an automobile electrical system via the cigarette lighter.

The recorder is an A.R. Vetter Company Model
C-4 analog instrumentation recorder. It has four FM channels, each with a bandwidth of dc to $100 \mathrm{~Hz}$. Three of the available FM channels (Channels 1, 2, and 3) are used for EEG signal recording. The fourth channel can be used either to record the electrocardiogram or as a system test channel. When Channel 4 is in the test mode, the fourth channel input is tied to circuit common and is used as a check on capstan speed stability (wow and flutter). A toggle switch is provided to select the mode of operation of the fourth channel. Four FM channels are created in the recorder package by frequency-multiplexing Channels 1 and 2 onto the left stereo channel of a standard Sony TC-161SD tape deck and multiplexing channels 3 and 4 onto the right stereo channel. The center frequency of channels 1 and 3 is $750 \mathrm{~Hz}$ and that of Channels 2 and 4 is $4,000 \mathrm{~Hz}$. These center frequencies are modulated by up to $\pm 60 \%$ by the data, thus minimizing flutter noise. While a recording is being made, the demodulation electronics are also operational, providing monitoring capability at the output connectors. These outputs have been internally connected to the Tektronics Model 211 oscilloscope through a rotary channel selector switch.

Since the input sensitivity of the A. R. Vetter recorder is not sufficient ( $2.0 \mathrm{~V} \mathrm{pp}$ max. sens.) for EEG signal acquisition, special amplifiers were designed for this purpose (see Figure 2). EEG signal amplification is performed in two stages. The first stage is an integrated-circuit differential-input instrumentation amplifier (Analog Devices Model AD521J) which is used to provide a gain of 100 with very high common mode rejection $(104 \mathrm{~dB}, \mathrm{dc}$ to $60 \mathrm{~Hz})$. The low-frequency noise level of this amplifier is also exceptionally good

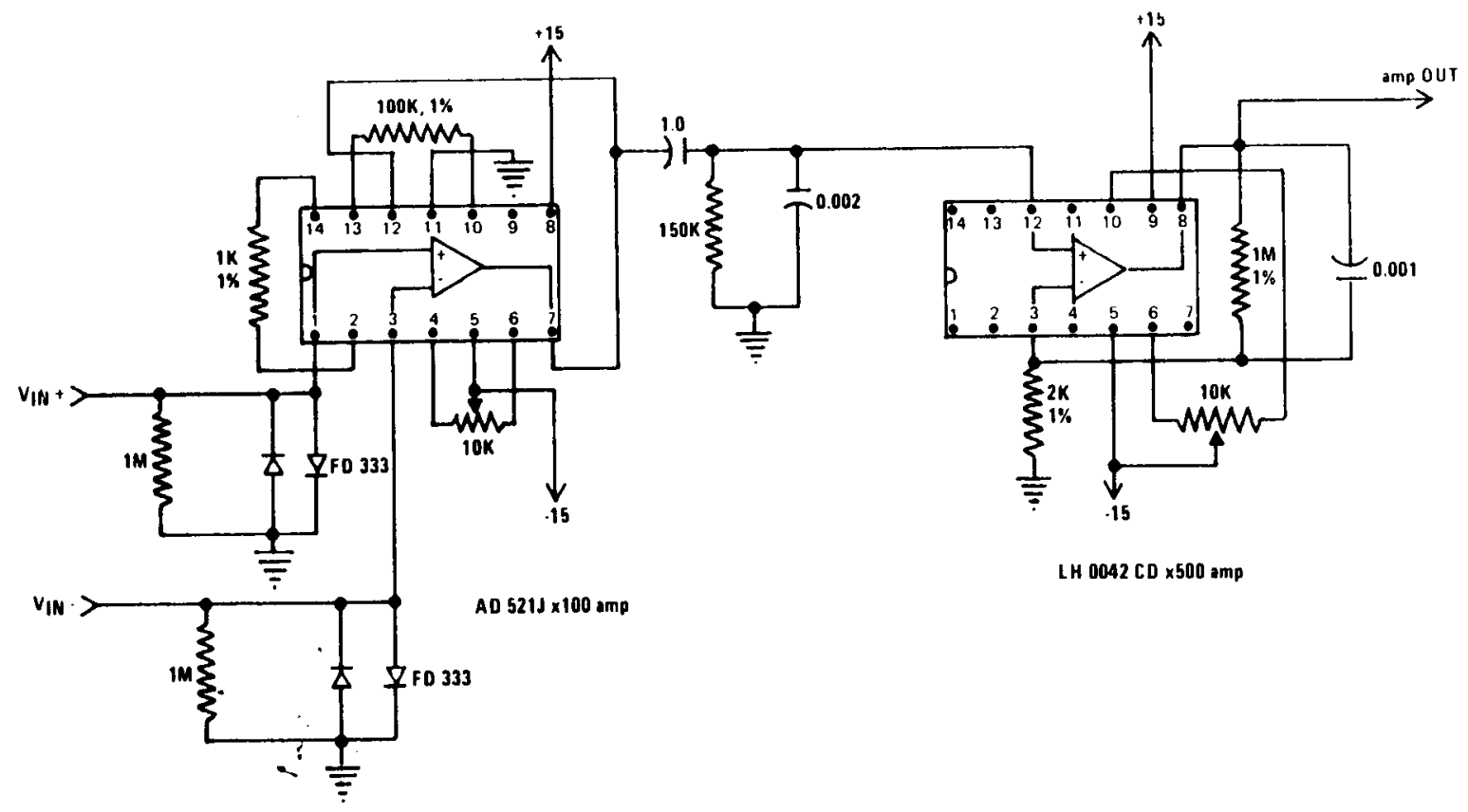


at 1.6 microV peak to peak from .1 to $10 \mathrm{~Hz}$. By limiting the gain to 100 in the first stage and ac coupling the output signal to the next stage, the rieed for offset adjustment controls is eliminated, since the expected electrode dc offset voltage is not large enough to drive the amplifier into saturation. A gain of 500 is provided by the second stage, an FET-input operational amplifier (National Semiconductor LH 0042CD) connected in the noninverting configuration. The exceedingly high input impedance of this amplifier permits the use of a relatively small coupling capacitor while achieving a low-frequency cutoff of about $1.0 \mathrm{~Hz}$.

The net gain of the EEG amplifier is 50,000 . This gain is sufficient to raise an 80-microV peak-to-peak EEG signal to $4.0 \mathrm{~V}$ pp. In order to obtain the full output level of $10 \mathrm{~V} \mathrm{pp}$ from the recorder with a $4.0-\mathrm{V}$ pp input signal, an additional gain of 2.5 was set on each channel of the recorder via the sensitivity controls. These controls were then potted, thus assuring a constant gain of 125,000 . Thus, the EEG signals obtained during playback can be converted to the true EEG levels at the electrodes simply by dividing the recorder output signal level by 125,000 .

The ECG amplifier (not shown) is identical to the EEG amplifier, with the exception that the gain of the second stage is reduced from 500 to 26 . Thus, with an ECG amplifier gain of 2,600 and the Channel 4 recorder sensitivity set for an additional gain of 2.5 , a 1.54 -
microV pp. ECG signal will result in a $10-\mathrm{V}$ pp recorder output level.

\section{PERFORMANCE}

The recorder will operate for $5 \mathrm{~h}$ from the power source before recharging. Cassette tape capacity is at present $60 \mathrm{~min} / \mathrm{side}$. System bandwidth is $1.100 \mathrm{~Hz}$, with the low-frequency cutoff determined by the preamp. Signal-to-noise ratio is $40 \mathrm{~dB}$. Power consumption is $23 \mathrm{~W}$. Crosstalk is less than noise level. There is no measurable change in recorded signal frequency down to $.1 \mathrm{~Hz}$ or in amplitude down to $.1 \mathrm{~V}$ when the input voltage is varied from 103 to $120 \mathrm{~V}$ ac. Total cost of all components including the Trav-Electric power source is under $\$ 2,500$. The power source weighs $13.2 \mathrm{~kg}$ (29 lbs) and the recorder $15 \mathrm{~kg}$ (33 lbs).

\section{REFERENCES}

BARLOW, J. S. A 16-channel cassette tape recorder system for clinical EEGs. Electroencephalography and Clinical Neurophysialogy, 1975, 38, 183-186.

IVES, J. R., \& Woods, J. F. Four-channel 24-hour cassette recorder for long-term EEG monitoring of ambulatory patients. Electroencephalography and Clinical Neurophysiology, 1975, 39, 88-92.

(Received for publication February 25, 1976; revision accepted April 16, 1976.) 